\title{
A gradient boosting approach to the Kaggle load forecasting competition
}

\author{
Souhaib Ben Taieb ${ }^{1}$ and Rob J Hyndman ${ }^{2}$ \\ ${ }^{1}$ Machine Learning Group, Department of Computer Science, Faculty of Sciences, Université Libre de Bruxelles \\ 2 Department of Econometrics and Business Statistics, Monash University, Clayton, VIC 3800, Australia
}

\begin{abstract}
We describe and analyse the approach used by Team TinTin (Souhaib Ben Taieb and Rob J Hyndman) in the Load Forecasting track of the Kaggle Global Energy Forecasting Competition 2012. The competition involved a hierarchical load forecasting problem for a US utility with 20 geographical zones. The available data consisted of the hourly loads for the 20 zones and hourly temperatures from 11 weather stations, for four and a half years. For each zone, the hourly electricity load for nine different weeks needed to be predicted without having the location of zones or stations. We used separate models for each hourly period, with component-wise gradient boosting to estimate each model using univariate penalised regression splines as base learners. The models allow for the electricity demand to change with time-of-year, day-of-week, time-of-day, and on public holidays, with the main predictors being current and past temperatures as well as past demand. Team TinTin ranked fifth out of 105 participating teams.
\end{abstract}

Keywords: short-term load forecasting, multi-step forecasting, additive models, gradient boosting, machine learning, Kaggle competition

\section{Introduction}

We participated in the Load Forecasting track of the Kaggle Global Energy Forecasting Competition 2012 organised by the IEEE working group on Energy Forecasting (WGEF) (Tao et al., 2013). Team TinTin (Souhaib Ben Taieb and Rob J Hyndman) ranked fifth out of 105 participating teams. The competition involved a hierarchical load forecasting problem. We were required to backcast and forecast hourly loads (in $\mathrm{kW}$ ) for a US utility with 20 geographical zones. Thus, 21 separate time series needed to be backcast and forecast: the 20 zonal level series, and the aggregate series.

Electricity demand is subject to a range of factors including weather conditions, calendar effect, economic activity and electricity prices. However we were required to use only temperatures and calendar information. The available data consisted of the hourly loads for the 20 zones, and hourly temperatures from 11 weather stations, from the first hour of 1 January 2004 to the sixth hour of 30 June 2008. We are unaware of the location of the 20 zones and the 11 weather stations; in particular, we do not know which stations are located in or near which zones. Consequently, our task was to find a model that takes temperature and calendar information as inputs, and predicts electricity load as the output.

We used a separate model for each hourly period ending up with 24 different models for one day since the electricity demand pattern is changing throughout the day. Each hourly model is estimated using component-wise gradient boosting (Bühlmann and Yu, 2003) with univariate penalised regression splines (Eilers and Marx, 1996) as base learners together with an automatic variable selection during the fitting process. The models can be cast in the framework of additive models allowing nonparametric and non-linear terms. The models allow for the electricity demand to change with time-of-year, day-of-week, time-of-day, and on public holidays. The main predictors were current and past temperatures (up to a week earlier) and past demand (up to a week earlier). 
Our approach has been influenced by the work of Fan and Hyndman (2012) who developed semiparametric additive models with penalised spline functions for forecasting electricity demand in various Australian states.

The paper is organised as follows. The next section presents the data analysis and preprocessing we performed. Section 3 describes our forecasting methodology, clarifying the differences between in-sample and out-of-sample weeks. Section 4 presents our model together with the gradient boosting algorithm. The proposed model is analysed in Section 5. Section 6 provides some discussion and conclusions.

\section{Data analysis and preprocessing}

Figure 1 shows the average demand for all zones during the period of the data. The average demand varied greatly across zones with Zone 18 having the highest demand levels and Zone 4 the least. By exploring the data, we noticed that Zones 3 and 7 contain identical data, and Zone 2 contains values that are exactly $92.68 \%$ of the demand values in Zones 3 and 7 . Also, Zone 10 has a big jump in demand in year 2008. Finally, Zone 9 contained very erratic demand patterns which did not seem to relate to the temperature values.

Electricity demand is subject to a wide variety of exogenous variables including calendar effects. Figure 2 shows that there is a clear time-of-year effect in the demand data with peaks in mean demand around February and July, and troughs in April/May and October. In others words, winter and summer are showing high demand while fall and spring have lower demand.

Boxplots of the demand by day of week are shown in Figure 3. While the day-of-week effect is relatively small, there is a drop in demand for the weekends. The average demand for each month

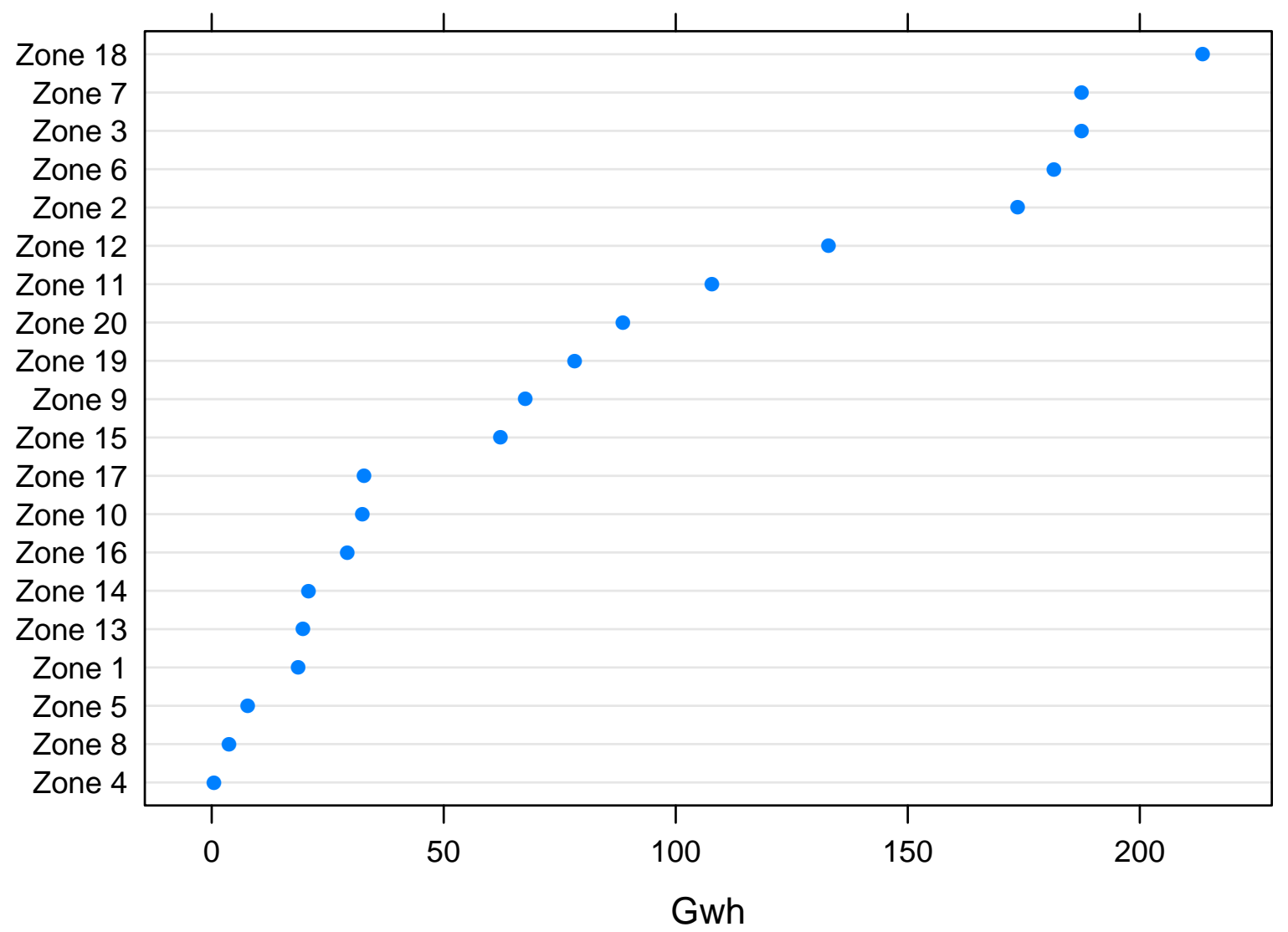

Figure 1: Average demand for each zone. 


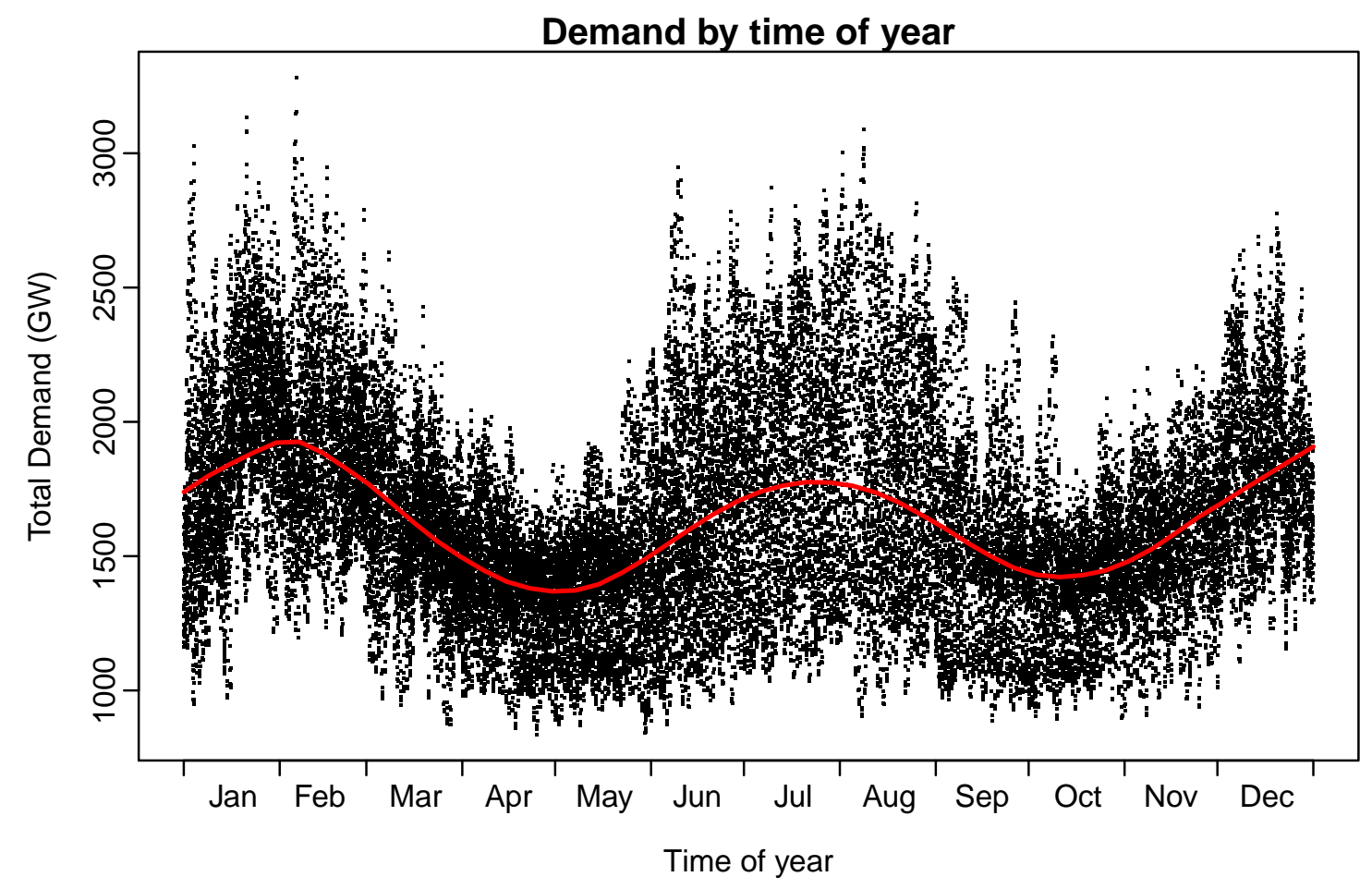

Figure 2: Total demand plotted against the time of year. The smoothed mean demand is shown as a red line.

and each day of the week is plotted in Figure 4. Because the day-of-week pattern is similar for all months, there is unlikely to be a strong interaction between day-of-week and time-of-year.

We look at the way demand changes with the time of day in Figures 5 and 6. Here hour 0 corresponds to $12 \mathrm{am}-1 \mathrm{am}$, hour 1 corresponds to $1 \mathrm{am}-2 \mathrm{am}$, and so on. The night-time pattern is similar for the two plots, but there is a difference during the working hours (8am-5pm).

Figure 7 shows demand in Zone 18 plotted against current temperature from station 9. There is a clear non-linear relationship, indicating current temperature is an important predictor of demand. For temperatures above $20^{\circ} \mathrm{C}$, air conditioning usage drives demand, whereas for temperatures below $15^{\circ} \mathrm{C}$, heating drives demand. Similar but weaker relationships are seen in plots against lagged temperatures. Due to thermal inertia in buildings, it is important to consider lagged temperatures as well as current temperatures in any demand forecasting model. Figure 8 shows current demand plotted against lagged demand for different lags. We can see the relationship between these variables due to the serial dependence within the demand time series.

Before developing any forecasting models, we pre-processed the data to avoid some potential problems. First, we removed leap days to have 365 days for each year. This avoided problems with uneven seasonal periods, and resulted in only a small loss of information. Second, we took a log transformation for the demand. This is to stabilise the variance of the time series across time. There were also some outliers and unusual features in the data which we corrected before proceeding.

We identified some outliers in site 8 for the temperature data, and replaced them with the data of the same period in the closest site in terms of Euclidean distance.

Zone 4 had some outliers in demand. We used Loess for fitting and then classified a point $y_{t}$ as an outlier if $y_{t}-\hat{y}_{t}>$ median $\left(y_{t}-\hat{y}_{t}\right)+k *$ MAD where $\hat{y}_{t}$ is the Loess fit of $y_{t}$, MAD is the mean absolute deviation and $k$ is chosen so the probability of an outlier is 0.002 under a normal distribution. Then, the outliers have been replaced by the mean of the data.

For Zone 10, there was a big jump in demand in year 2008. We computed the mean before the jump and the mean after the jump on the log-transformed data. Then we took the difference and removed the jump in 2008. For the final forecasts, we restored the jump into the forecasts. 


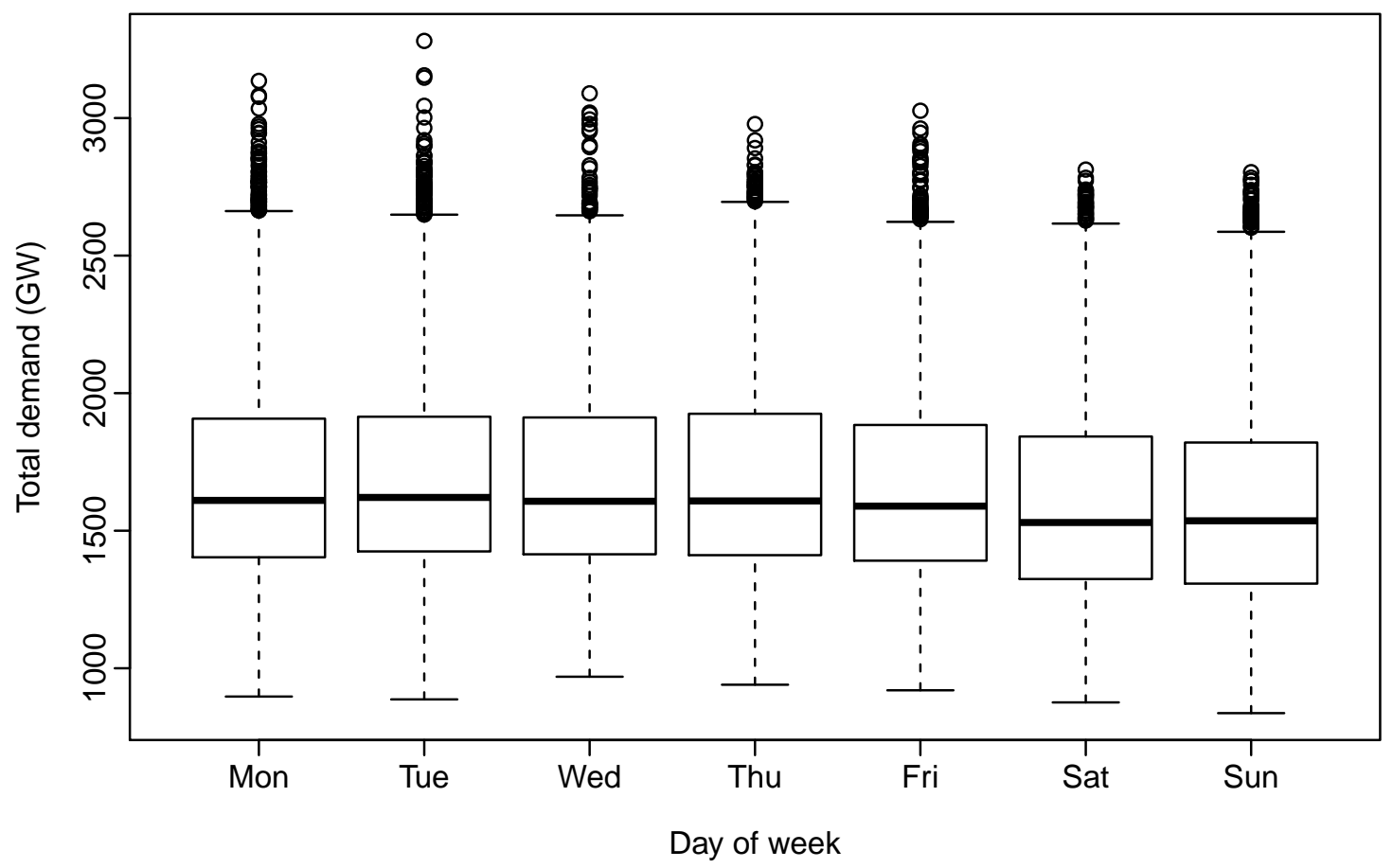

Figure 3: Boxplots of total demand by day of week.

Mean demand by month and day of week

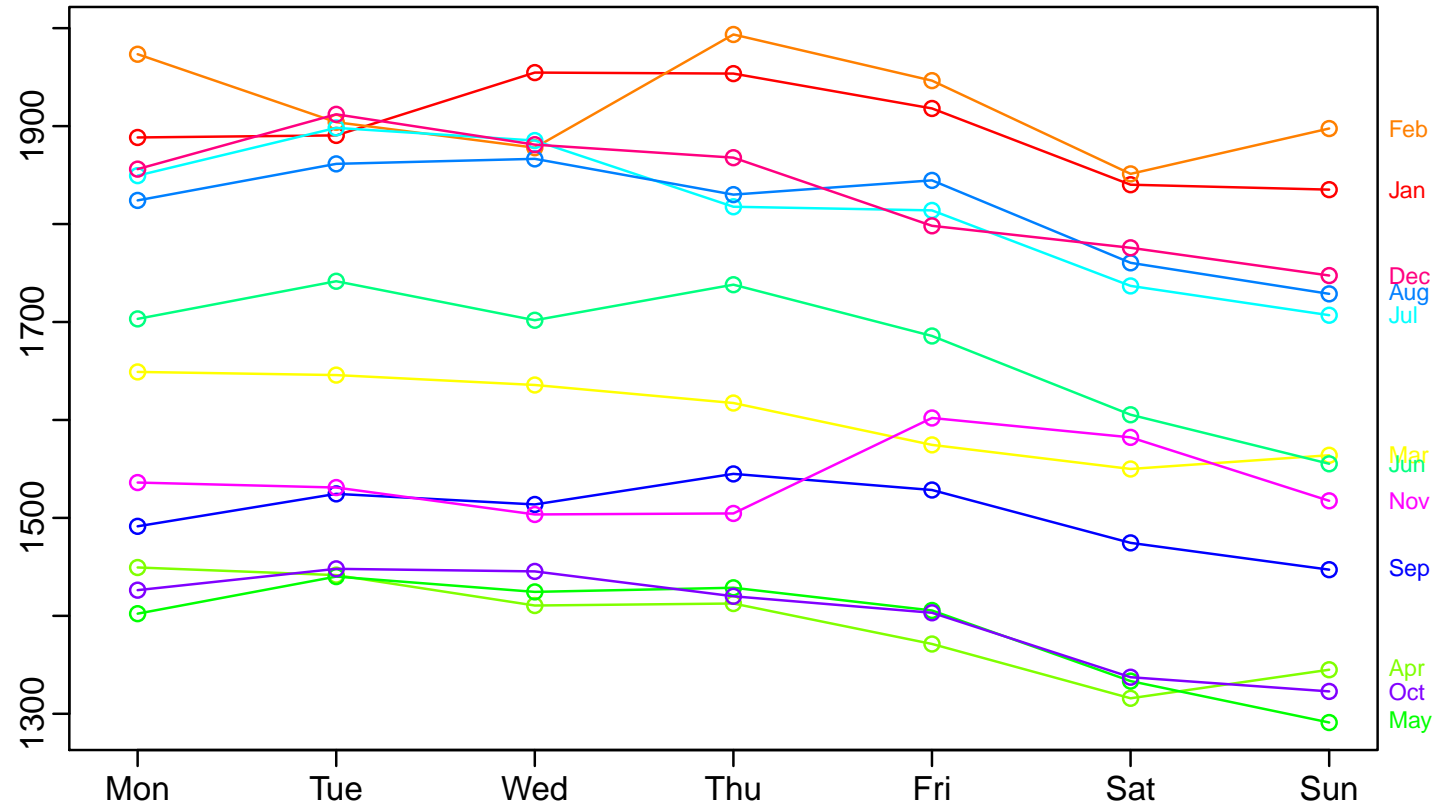

Figure 4: Average total demand (GW) by month and day of week. 


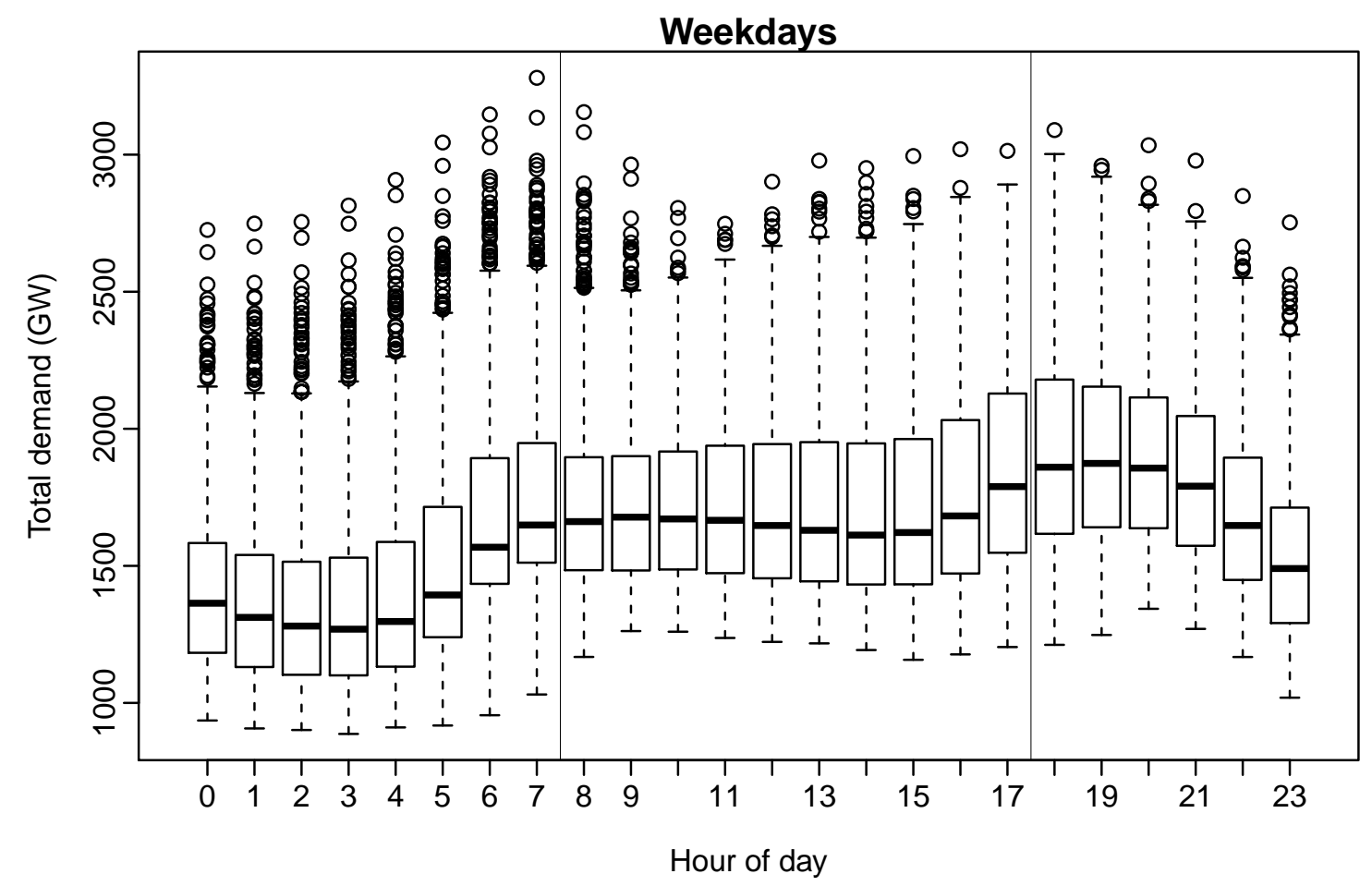

Figure 5: Boxplots of demand by time of day for Monday-Friday.

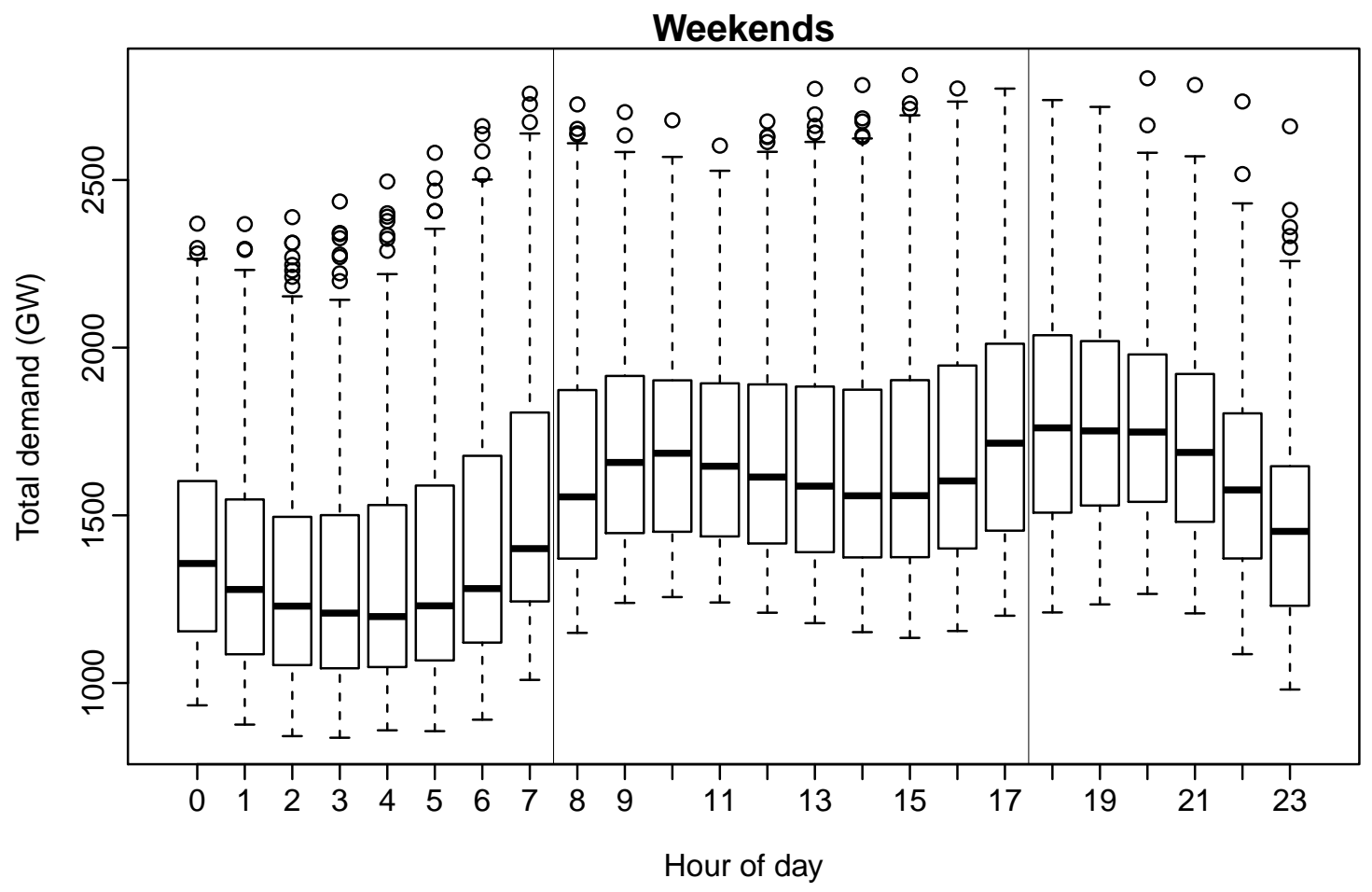

Figure 6: Boxplots of demand by time of day for Saturday-Sunday. 


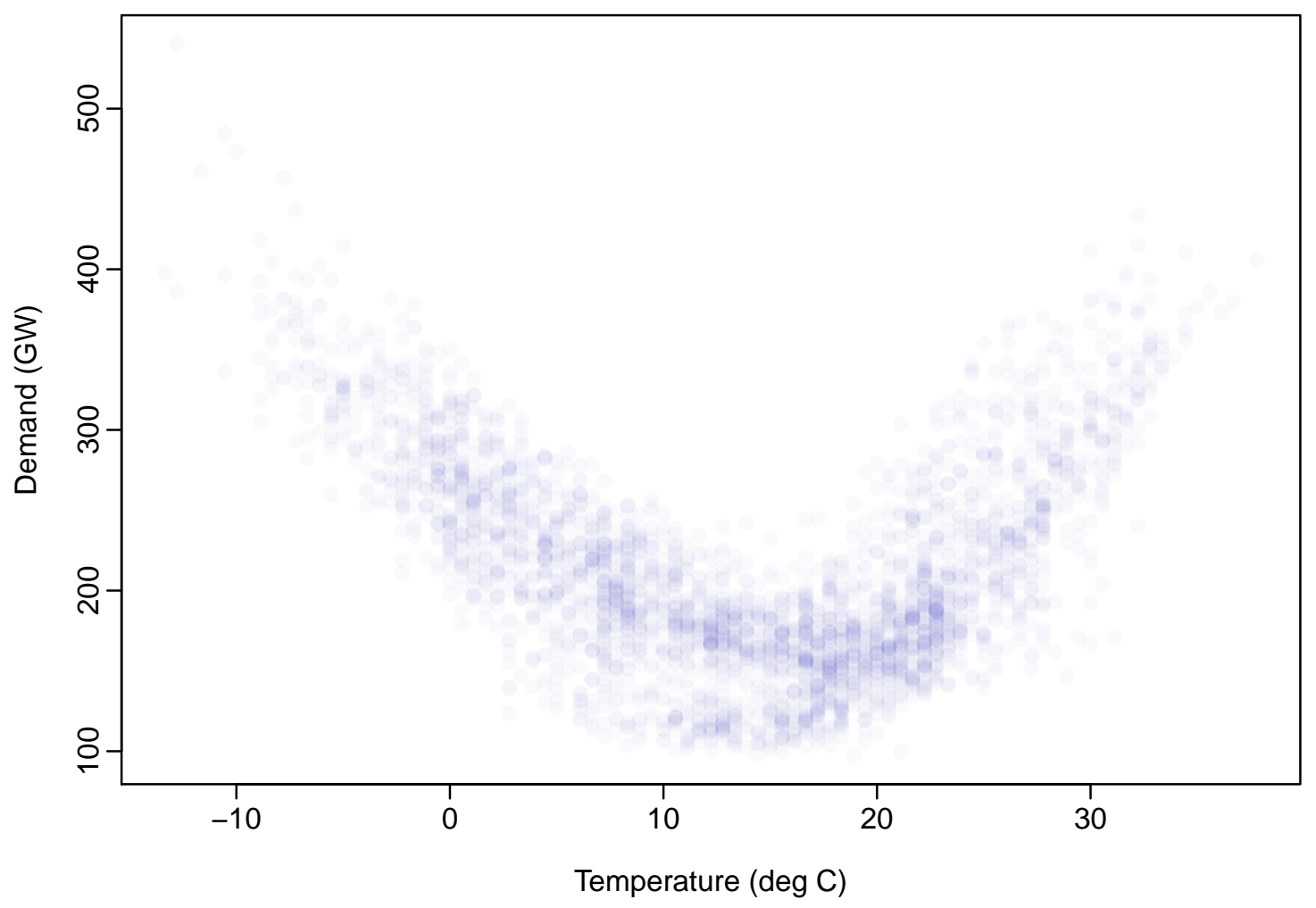

Figure 7: Hourly demand (GW) plotted against temperature (degrees Celsius) for Zone 18 and station 9.
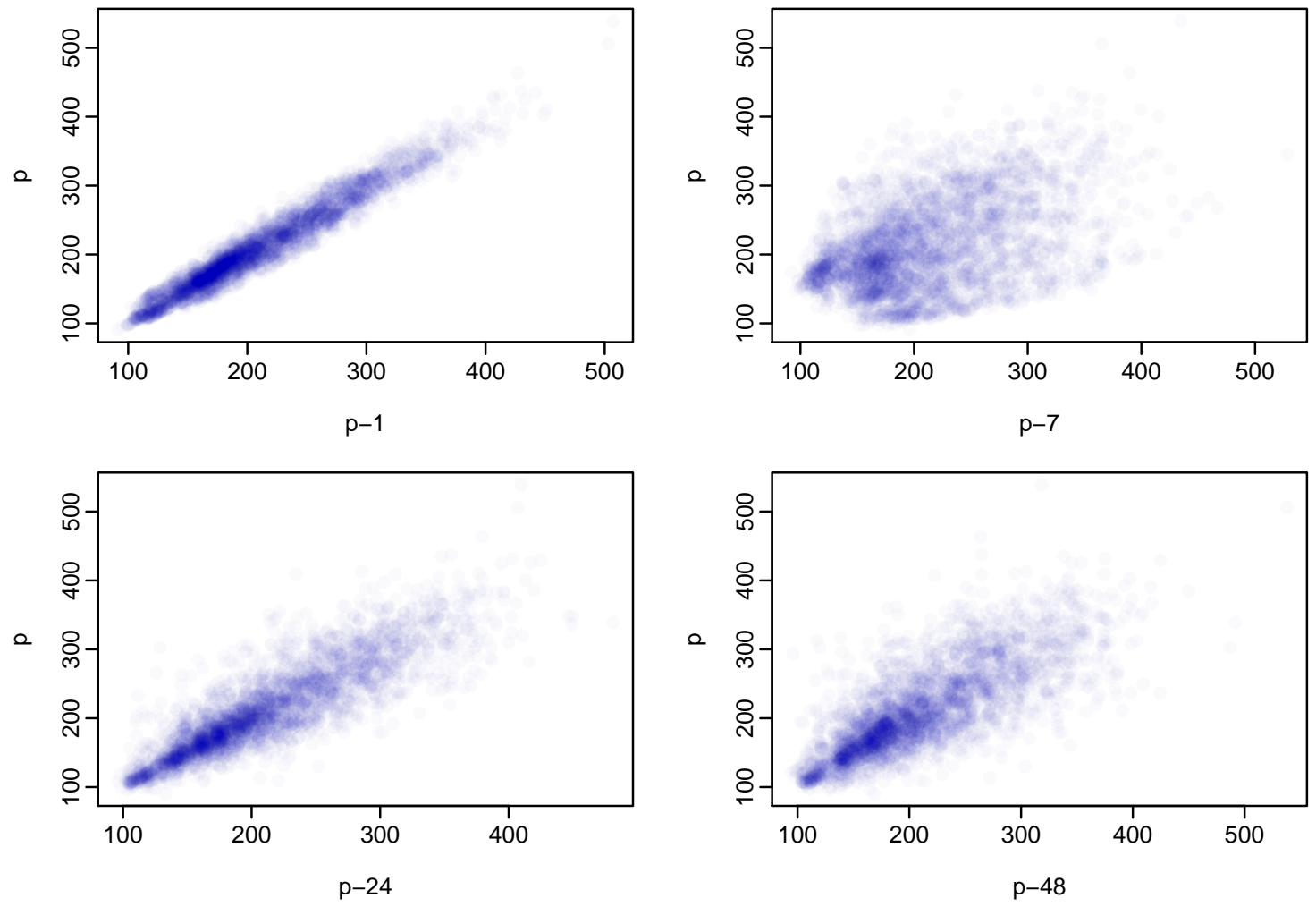

Figure 8: Current demand plotted against lagged demand for different lags for Zone 18. 


\section{Forecasting methodology}

The competition involved a hierarchical load forecasting problem with 20 zonal level series, and the aggregate series. We used a bottom-up approach, that is we forecast each zone independently and then we take the sum of the 20 zonal forecasts to obtain forecasts for the aggregate. For each of the twenty zones, we were required to backcast the demand for eight in-sample weeks for which the temperature at various sites was provided.

Because we do not know which temperature site corresponds to which zone, the temperatures from all sites are potential predictors in each model. We used a testing week (the last week of the available data) to determine which sites to use for each zone. Figure 9 gives the root mean squared error (RMSE) obtained on the testing week using the real temperature (in blue) and the forecasted temperature (in red) from the eleven sites for ten zones. We can see that the difference in forecasts obtained using the real temperature (in blue) at different sites can be huge. Consequently, when forecasting the eight in-sample weeks, we use, for each zone, the site which minimises the error over the testing week.

For the eight in-sample weeks, data was available before and after the week for demand and during the week for the temperature. The data after each of the in-sample weeks is also useful for predicting the demand values during the in-sample weeks (although this is not possible in real forecasting operations). In order to use this data, we fitted two forecasting models. The first model was estimated in the usual way using data available up to the start of the in-sample week. The second model reversed the time ordering of the data so we were back-casting using data available after the end of the in-sample week. We expect the forecasts to do best at the beginning of the week, and the backcasts to do best at the end of the week, because they involve data closer to the days being predicted. So, after estimating the two sets of models, we took a weighted combination of both sets of forecasts to produce the final forecasts. More precisely, if we denote $\hat{y}_{t+h}^{(\mathrm{F})}$, the forward forecasts and $\hat{y}_{t+h}^{(\mathrm{B})}$, the backward forecasts with $h=[1, \ldots, 168]$, then the final forecasts are given by

$$
\hat{y}_{t+h}=w_{h} \hat{y}_{t+h}^{(\mathrm{F})}+\left(1-w_{h}\right) \hat{y}_{t+h}^{(\mathrm{B})}
$$

where $w_{h}=\operatorname{sigmoid}\left(-7+\frac{14 * h}{168}\right)$ and $\operatorname{sigmoid}(x)=1 /\left(1+e^{-x}\right)$ is the sigmoid function.

Forecasts were also required for one out-of-sample week without temperature data. In contrast with the in-sample weeks, temperatures were not provided for the out-of-sample week. So we had to forecast the temperatures for this week and used these when forecasting demand during that week. We used the average temperature at the same period across the years as forecasts, shown in Figure 10. Differences between out-of-sample forecasts obtained using different temperature sites are not as large as for the in-sample weeks as can be seen in Figure 9 (see red bars). This is because we do not have actual temperatures in that week, and our forecast temperatures have a smaller range than the actual temperatures. So, for out-of-sample forecasts, we average the forecasts obtained from the three best temperature sites when forecasting demand in order to reduce the variance of the forecasts. These sites are also shown in Figure 9.

Since the demand patterns varies greatly during the year and for computational convenience, we did not use the whole demand data to estimate our models. Instead, for each of the available years, we used part of the data around the week to be forecast. More precisely, we computed the average temperature for the week to be forecast and, for each year, we select the 12 consecutive weeks around this week which have the closest average temperature. Then we filtered the demand data by keeping these 12 consecutive weeks of each year. For the out-of-sample week, for which we do not have the average temperature for the week to be forecast, we used the average temperature of the previous week to perform the same calculations.

The above procedures was followed for all zones except zones 2, 7 and 9. Zones 3 and 7 contain identical data, and Zone 2 contains values that are exactly $92.68 \%$ of the demand values in Zones 3 

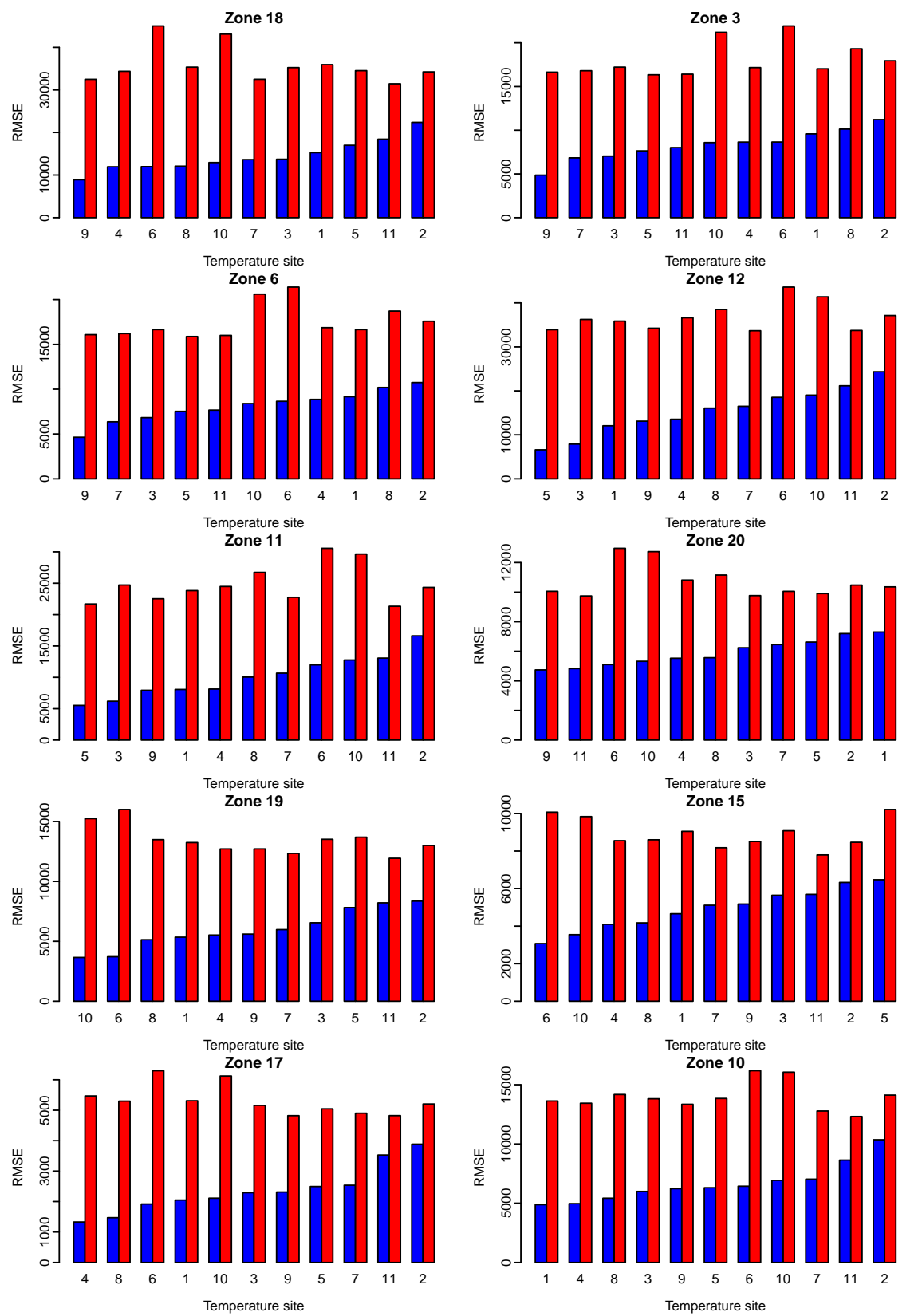

Figure 9: Root mean square error (RMSE) over the testing week using real temperature (in blue) and forecasted temperature (in red). The sites are ranked according to the RMSE when using real temperature.

and 7. Consequently, we do not fit separate models for Zones 2 and 7, instead we use the forecasts from Zone 3 to compute forecasts for Zones 2 and 7. And for Zone 9, we used the average of the same hour for every day of the week over the entire data set to obtain forecasts for each period as we didn't find any temperature-related patterns.

\section{Forecasting model}

One of the earliest electricity forecasting competitions was won by Ramanathan et al. (1997) using a separate regression model for each hour of the day. This idea has subsequently been used by Fay et al. (2003); McSharry et al. (2005); Fan and Chen (2006) and Fan and Hyndman (2012).

We follow the same approach and have a separate model for each hour of the day. These models are used recursively to produce the 168 required forecasts ( 24 hours multiplied by 7 days). That is, 

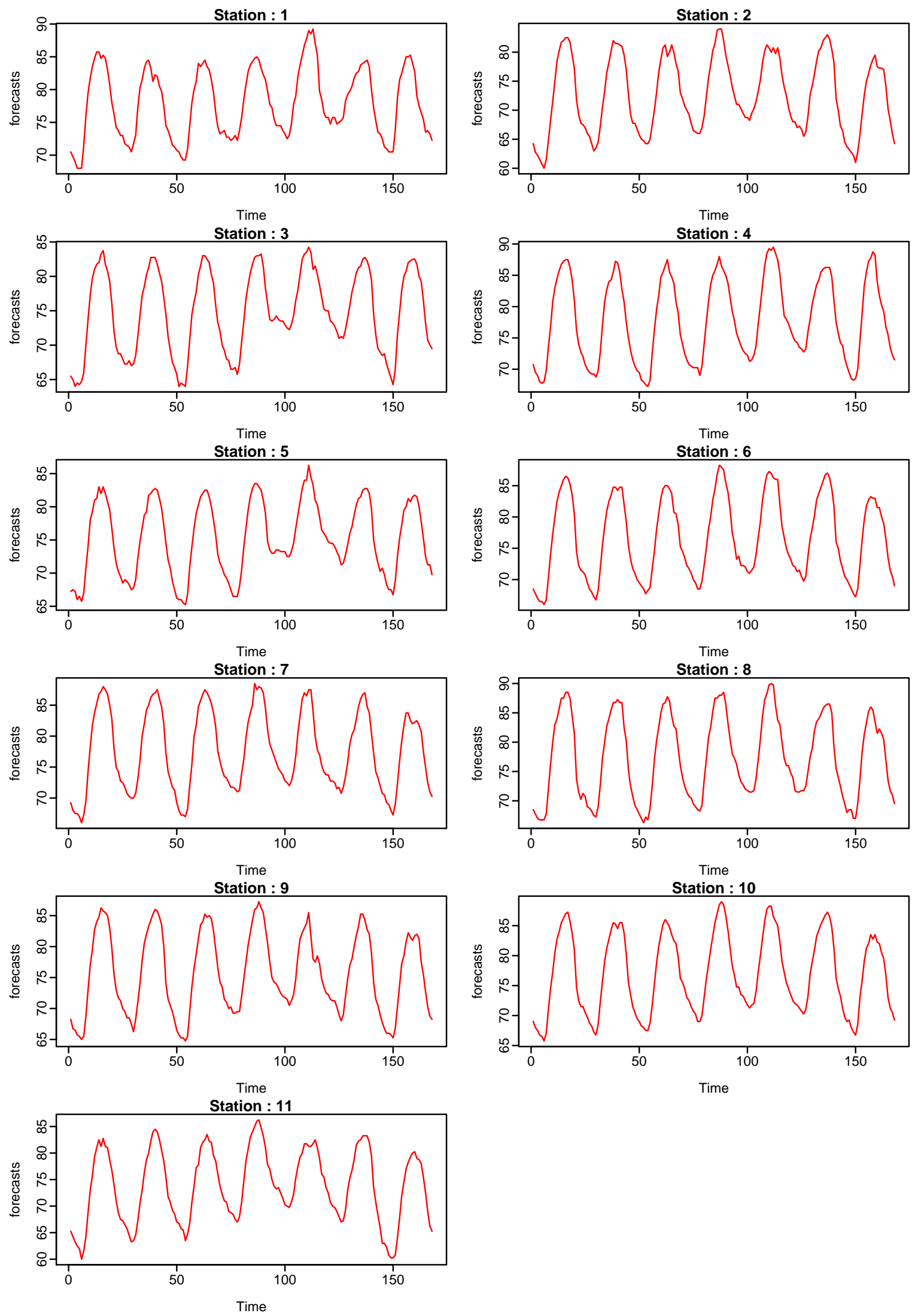

Figure 10: Forecasts of temperature for the eleven stations. 
we first produce the forecasts of the next day and then, use them as inputs to make forecasts for the day after. We also added the observations from the previous hour and the next hour in order to have more data. That is, when estimating the model for hour $p$, we used data from hours $p-1, p$ and $p+1$.

We fit a model of this form for each hour of the day, for each zone, and for each of the nine weeks to be forecast. We ended up with $24 \times 17 \times 9=3672^{1}$ models to estimate with datasets having approximately 1000 observations and 43 input variables. The main predictors of the models are current and past temperatures (up to a week earlier) and past demand (up to a week earlier). In addition, the models allow for the demand to change with time-of-year, day-of-week, time-of-day, and on public holidays.

Each regression uses a nonparametric additive model with penalised regression splines. Componentwise gradient boosting is used to estimate each model including variable selection during the fitting process. Both aspects are detailed in the following sections.

\subsection{Non-parametric additive models}

We use nonparametric additive models for forecasting electricity demand. These models are in the regression framework but with some non-linear relationships. In particular, the proposed models allow nonlinear and nonparametric terms using the framework of additive models (Hastie and Tibshirani, 1995).

The demand in hour $p$ on day $t$ is modelled with

$$
y_{t, p}=c_{p}(t)+f_{p}\left(y_{t, p}\right)+g_{p}\left(z_{t, p}\right)+\varepsilon_{t},
$$

where

- $y_{t, p}$ denotes the demand on day $t$ in hour $p$;

- $c_{p}(t)$ models all calendar effects (including time-of-year, day-of-week, holidays, etc.);

- $f_{p}\left(y_{t, p}\right)$ models the effect of recent demand variables where $y_{t, p}$ is a vector of past demands, prior to hour $p$ on day $t$;

- $g_{p}\left(z_{t, p}\right)$ models the temperature effects where $z_{t, p}$ is a vector of recent temperatures variables at one of the temperature sites, prior to and including hour $p$ on day $t$; and

- $\varepsilon_{t}$ denotes the model error at time t.

We fit separate models of this form for each zone and all in-sample and out-of-sample weeks.

\subsubsection{Calendar effects}

The calendar effects term, $c_{p}(t)$, includes annual, weekly and daily seasonal patterns as well as public holidays.

- The day-of-week effect was modelled with a factor variable, taking a different value for each day of the week.

- The holiday effect was modelled with a factor variable, taking value zero on a non-work day ${ }^{2}$, some non-zero value on the day before a non-work day and a different value on the day after a non-work day.

- The time-of-year effect was estimated using a simple first-order Fourier approximation (one sine and one cosine variables). A more complicated time-of-year effect was not necessary as each model only included data within a 12 week period.

\footnotetext{
${ }^{1}$ There are only 17 zones to be modelled because of the relationship between Zones 2, 3 and 7, and because we use a different approach for Zone 9.

${ }^{2}$ We used the US federal holidays as listed in US Office of Personnel Management website: http://www.opm.gov/ Operating_Status_Schedules/fedhol/2008.asp
} 


\subsubsection{Temperature effects}

Due to thermal inertia in buildings, it is important to consider lagged temperatures as well as current temperatures in any demand forecasting model. The function $g_{p}\left(z_{t, p}\right)$ models the effects of recent temperatures on the aggregate demand where $z_{t, p}$ includes

- The current temperature and temperatures from the preceding 12 hours, and for the equivalent hour on each of the previous two days;

- The minimum and maximum temperature in both the last 24 hours and the previous day;

- The average temperature for the previous day, the day preceding the previous day and the last seven days.

Recall that when forecasting the eight in-sample weeks, the temperatures from the site which gave the best forecasts (on the testing week) for each zone were used. When forecasting the out-ofsample week, the demand forecasts obtained using the best three temperature sites were averaged.

\subsubsection{Lagged demand effects}

We incorporate recent demand values into the model via the function $f_{p}\left(y_{t, p}\right)$ where $y_{t, p}$ includes

- Lagged demand for each of the preceding 12 hours, and for the equivalent hour in each of the previous two days. For example, when predicting demand at $4 \mathrm{pm}$, we use lagged demand from 4 am to $3 \mathrm{pm}$, as well as lagged demand at $4 \mathrm{pm}$ on the preceding two days.

- The minimum and maximum demand in the last 24 hours.

- The average demand for the last seven days.

By doing this, the serial correlations within the demand time series can be captured within the model, and the variations of demand level throughout the time can be embedded into the model as well.

Finally, we did not necessarily use all the previous predictors in each model, but these were all candidate variables in our models. The process of selecting variables is described in the next section together with the gradient boosting algorithm.

\subsection{Component-wise gradient boosting with penalised splines}

Boosting is a prediction algorithm stemming from the machine learning literature based on the idea of creating an accurate learner by combining many so-called "weak learners". Since its inception in 1990 (Schapire, 1990; Freund, 1995; Freund and Schapire, 1996), Boosting has attracted much attention due to its excellent prediction performance in a wide range of applications both in the machine learning and statistics literatures (Schapire and Freund, 2012). The improved performance of boosting seems to be associated with its resistance to overfitting which is still under investigation (Mease and Wyner, 2008; Mease, 2008).

The gradient descent view of boosting (Friedman and Hastie, 2000; Friedman, 2001) has connected boosting to the more common optimisation view of statistical inference while previous work focused on bounding the generalisation error via the VC dimension and the distribution of so-called margins (Schapire et al., 1998). Gradient boosting (Friedman, 2001) interprets boosting as a method for function estimation from the perspective of numerical optimisation in function space.

Bühlmann and Yu (2003) developed component-wise gradient boosting to handle high-dimensional regression problems by selecting one variable at each iteration of the boosting procedure. We refer to Schapire and Freund (2012) and Bühlmann and Hothorn (2007) for a general overview on boosting.

For all our regression tasks, we used component-wise gradient boosting (Bühlmann and Yu, 2003; Bühlmann, 2006) with penalised regression splines (P-splines) (Eilers and Marx, 1996). By doing so, we take advantage of the good performance and the automatic variable selection of the boosting algorithm. In addition, P-splines allow us to have smooth estimation of the demand. We now provide more details about the procedure we implemented.

Notice that expression (1) can be rewritten as

$$
y_{t, p}=F_{p}\left(\boldsymbol{x}_{t}\right)+\varepsilon_{t, p}
$$


where $\boldsymbol{x}_{t}=\left[t, y_{t, p}, z_{t, p}\right]$ contains all potential predictors to be considered in the model. Our goal is to estimate the function $F_{p}: \mathbb{R}^{d} \rightarrow \mathbb{R}$ for a given loss function. Since, the forecasting accuracy for the competition was evaluated by weighted root mean square error, we used the quadratic loss function. Estimation of the function $F_{p}$ based on a sample dataset $\left\{\left(y_{t, p}, \boldsymbol{x}_{t}\right)\right\}_{t=1}^{T}$ reduces to minimising

$$
\hat{F}_{p}=\underset{F_{p}}{\operatorname{argmin}} \frac{1}{T} \sum_{t=1}^{T}\left(y_{t, p}-F_{p}\left(\boldsymbol{x}_{t}\right)\right)^{2} .
$$

Gradient boosting estimates $F_{p}$ in a stagewise manner. We let $\hat{F}_{p}^{(m)}\left(\boldsymbol{x}_{t}\right)$ denote the estimation of $F_{p}$ at the $m$ th stage, where $m=0,1, \ldots, M$. The process begins with $\hat{F}_{p}^{(0)}(\boldsymbol{x})=\bar{y}_{p}$ where $\bar{y}_{p}$ is the mean demand for hour $p$. Then the model is updated using

$$
\hat{F}_{p}^{(m)}\left(\boldsymbol{x}_{t}\right)=\hat{F}_{p}^{(m-1)}\left(\boldsymbol{x}_{t}\right)+v h_{m}\left(\boldsymbol{x}_{t} ; \hat{\boldsymbol{\theta}}_{m}\right),
$$

where $h_{m}\left(\boldsymbol{x}_{t} ; \hat{\boldsymbol{\theta}}_{m}\right)$ is the weak learner estimate at the $m$ th stage with parameters $\hat{\boldsymbol{\theta}}_{m}$ and $v \in[0,1]$ is a shrinkage parameter. Gradient boosting with the quadratic loss is also called $\mathrm{L}_{2}$ Boost (Bühlmann and $\mathrm{Yu}, 2003)$.

Let us note $\hat{h}_{m}\left(\boldsymbol{x}_{t}\right)$ as a shorthand for $h_{m}\left(\boldsymbol{x}_{t} ; \hat{\boldsymbol{\theta}}_{m}\right)$. Given an estimation $\hat{F}_{p}^{(m-1)}$, each additional term $\hat{h}_{m}\left(\boldsymbol{x}_{t}\right)$ is obtained by computing the negative gradient

$$
u_{t, p}^{m}=-\left.\frac{\frac{1}{2} \partial\left(y_{t, p}-F\left(\boldsymbol{x}_{t}\right)\right)^{2}}{\partial F\left(\boldsymbol{x}_{t}\right)}\right|_{F(\boldsymbol{x})=F_{p}^{(m-1)}(\boldsymbol{x})}=\left(y_{t, p}-F_{p}^{(m-1)}\left(\boldsymbol{x}_{t}\right)\right)
$$

which gives the steepest descent direction. Then, a regression is applied on $\left\{u_{t, p}^{m}, \boldsymbol{x}_{t}\right\}_{t=1}^{T}$ by the weak learner, i.e.

$$
\hat{\boldsymbol{\theta}}_{m}=\underset{\boldsymbol{\theta} \in \Theta}{\operatorname{argmin}} \sum_{t=1}^{T}\left[u_{t, p}^{m}-h_{m}\left(\boldsymbol{x}_{t} ; \boldsymbol{\theta}\right)\right]^{2}
$$

In other terms, $\hat{h}_{m}\left(\boldsymbol{x}_{t}\right)$ is selected to best predict the residuals from the previous model $F_{p}^{(m-1)}\left(\boldsymbol{x}_{t}\right)$.

Finally, the solution is given by

$$
\hat{F}_{p}\left(\boldsymbol{x}_{t}\right)=\hat{F}_{p}^{(M)}\left(\boldsymbol{x}_{t}\right)=\hat{h}_{0}\left(\boldsymbol{x}_{t}\right)+\sum_{m=1}^{M} v \hat{h}_{m}\left(\boldsymbol{x}_{t}\right)
$$

where the estimation of $\hat{F}_{p}$ is continuously improved by an additional component (or boost) $v \hat{h}_{m}$ at stage $m$ and the hyperparameter $M$ prevents overfitting by limiting the number of components.

From expression (3), we can see that the boosting procedure depends on two hyperparameters: $v$, the shrinkage parameter and $M$, the number of components (or number of stages). The value of $v$ affects the best value for $M$, i.e. decreasing the value of $v$ requires a higher value for $M$. Since they can both control the degree of fit, we should ideally find the best value for both of them by minimising some model selection criterion. However, Friedman (2001) shows that small values of $v$ are better in terms of less overfitting of the boosting procedure. Hence, there is only one hyperparameter remaining for which the best value need to be selected (Bühlmann and Yu, 2003).

In expression (2), the weak learner is estimating the model parameters using all the predictors simultaneously A better procedure can be used when having many predictors: component-wise gradient boosting (Bühlmann and Yu, 2003; Bühlmann, 2006). The key idea is to use the weak learner with one variable at a time and select the one with the largest contribution to the fit. In other terms, 
expression (2) is replaced by the following procedure:

1. $\hat{\boldsymbol{\theta}}_{m}^{(k)}=\underset{\boldsymbol{\theta} \in \Theta}{\operatorname{argmin}} \sum_{t=1}^{T}\left[u_{t, p}^{m}-h_{m}\left(x_{k t} ; \boldsymbol{\theta}\right)\right]^{2}$ where $x_{k t}$ is the $k$ th variable of $\boldsymbol{x}_{t}$ and $k=1, \ldots, d$.

2. $k_{m}=\underset{k \in 1, \ldots, d}{\operatorname{argmin}} \sum_{t=1}^{T}\left[u_{t, p}^{m}-h_{m}\left(x_{k t} ; \hat{\theta}_{m}^{(k)}\right)\right]^{2}$.

3. Use $\hat{\boldsymbol{\theta}}_{m}^{\left(k_{m}\right)}$ at the $m$ th stage.

Then, the final solution is expressed as

$$
\hat{F}_{p}\left(x_{t}\right)=\hat{h}_{0}\left(x_{k_{0}} t\right)+\sum_{m=1}^{M} v \hat{h}_{m}\left(x_{k_{m} t}\right)
$$

where $\hat{h}_{m}$ is function of the $k_{m}$ th predictor.

In our implementation, we used P-splines with 20 equally spaced knots and four degrees of freedom for the weak learner $h_{m}\left(x_{k_{m}}\right)$ terms. For the hyperparameters values, we set the value of $v$ to 0.15 and the maximum number of components (or stages) $M$ to 500. Our implementation of the model depended on the mboost package for $\mathrm{R}$ (Hothorn et al., 2012). We used the gamboost function with the following values for the mboost_control parameters: nu=0.15 and mstop $=500$. For the base learners, we used the bbs function for numerical variables and the bols function for factor variables. Finally, we select the best number of stages $M(M \in\{1, \ldots$, mstop $\})$ using the cvrisk function with 5 -fold cross-validation.

\section{Model analysis}

In this section, we analyse and interpret the results of the proposed forecasting model to shed light about its attractive features.

Figure 11 gives the root mean squared error (RMSE) obtained for the different zones on the testing week. Zones that have higher errors are also zones with high average demand (see Figure 1). This suggests that zones with high average demand will carry a higher weight in the final error.

Figure 12 gives the true hourly demand together with the fitted values for an increasing number of boosting iterations. Recall that gradient boosting is a stagewise fitting procedure which depends on an hyperparameters $M$ (the number of boosting iterations), as shown in formula (4). We can see that the first iterations significantly reduce the error of the model while the final iterations are less and less contributing to the model. This confirms the theoretical analysis performed in (Bühlmann and $\mathrm{Yu}, 2003$ ) where the authors proof that the bias decreases exponentially fast and the variance increases with exponentially diminishing terms as the number of boosting iteration $M$ increases.

One of the attractive feature of the component-wise boosting algorithm is the automatic variable selection induced by the procedure at each iteration. That is, among all the potential predictors which are given in Table 1, few will be selected and contribute to each hourly model. See Section 4.1 for a more detailed description of the different predictors.

Note that the final solution of the boosting procedure, given in formula (4), can be rewritten as

$$
\hat{F}_{p}\left(\boldsymbol{x}_{t}\right)=\sum_{m=0}^{M} v \hat{h}_{m}\left(x_{k_{m} t}\right)=\sum_{k \in\{1, \ldots, d\}} \underbrace{\sum_{\left.m: k_{m}=k\right\}} v \hat{h}_{m}\left(x_{k_{m} t}\right)}_{\hat{H}\left(x_{k t}\right)}=\sum_{k \in\{1, \ldots, d\}} \hat{H}\left(x_{k t}\right),
$$




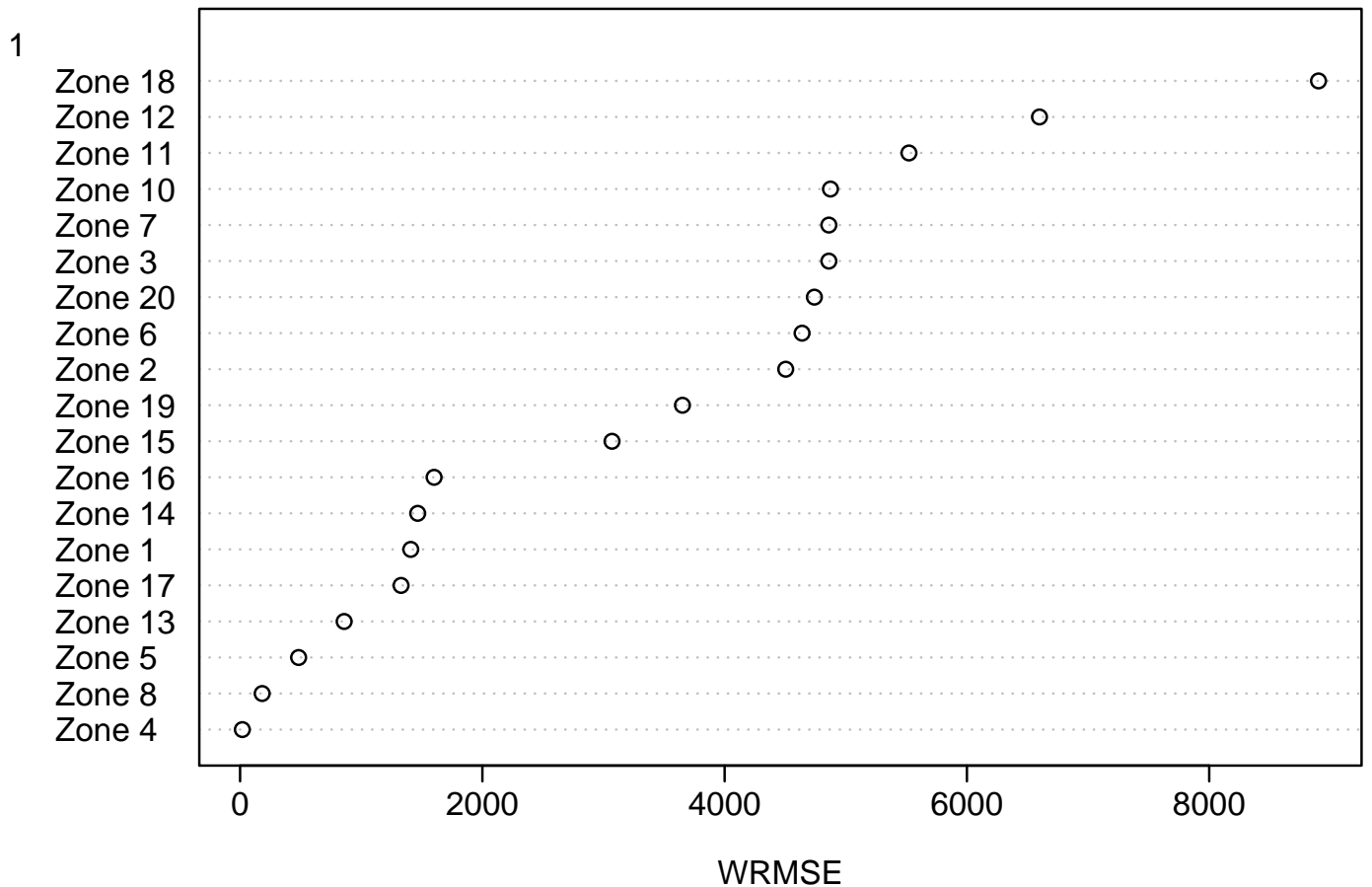

Figure 11: Root mean squared error (RMSE) obtained for each zone on the testing week.

\begin{tabular}{|c|c|c|c|c|c|c|c|}
\hline Id & Variable & Id & Variable & Id & Variable & Id & Variable \\
\hline 1 & day of the week & 12 & demand $[\mathrm{t}-8]$ & 23 & temp.[t+h-2] & 34 & temp. $[\mathrm{t}+\mathrm{h}-13]$ \\
\hline 2 & holiday & 13 & demand $[t-9]$ & 24 & temp. $[t+h-3]$ & 35 & temp. $[\mathrm{t}+\mathrm{h}-25]$ \\
\hline 3 & time of year $(\sin )$ & 14 & demand $[t-10]$ & 25 & temp. $[\mathrm{t}+\mathrm{h}-4]$ & 36 & temp. $[\mathrm{t}+\mathrm{h}-49]$ \\
\hline 4 & time of year (cos) & 15 & demand $[t-11]$ & 26 & temp. $[t+h-5]$ & 37 & min. temp. (prev 1$)$ \\
\hline 5 & demand $[t-1]$ & 16 & demand $[t-12]$ & 27 & temp. $[t+h-6]$ & 38 & min. temp. (prev 2) \\
\hline 6 & demand $[t-2]$ & 17 & demand $[t+h-25]$ & 28 & temp. $[\mathrm{t}+\mathrm{h}-7]$ & 39 & max. temp. (prev 1) \\
\hline 7 & demand $[t-3]$ & 18 & demand $[t+h-49]$ & 29 & temp. $[\mathrm{t}+\mathrm{h}-8]$ & 40 & max. temp. (prev 2) \\
\hline 8 & demand $[t-4]$ & 19 & min. demand (prev 1) & 30 & temp.[t+h-9] & 41 & avg. temp. (prev 1-7) \\
\hline 9 & demand $[t-5]$ & 20 & max. demand (prev 1) & 31 & temp. $[\mathrm{t}+\mathrm{h}-10]$ & 42 & avg. temp. (prev 1) \\
\hline 10 & demand $[t-6]$ & 21 & avg. demand (prev 1-7) & 32 & temp. $[\mathrm{t}+\mathrm{h}-11]$ & 43 & avg. temp. (prev 3) \\
\hline 11 & demand [t-7] & 22 & temp. $[\mathrm{t}+\mathrm{h}-1]$ & 33 & temp. $[\mathrm{t}+\mathrm{h}-12]$ & - & - \\
\hline
\end{tabular}

Table 1: Description of all potential predictors. Forecasts are from demand[t-1] to demand $[\mathrm{t}+\mathrm{h}-1]$ where $h \in\{1, \ldots, 24\}$.

where $\hat{H}\left(x_{k_{m} t}\right)$ is the relative contribution of the variable $k$ to the final model and $d$ is the number of initial predictors (the dimensionality of $\boldsymbol{x}_{t}$ ).

Let us define the model without the effect of predictor $j$ as

$$
\hat{F}_{p}^{(-j)}\left(\boldsymbol{x}_{t}\right)=\sum_{k \in\{1, \ldots, d\} \backslash\{j\}} \hat{H}\left(x_{k t}\right),
$$

and the corresponding squared error as

$$
E^{(-j)}=\sum_{t=1}^{T}\left(y_{t}-F_{p}^{(-j)}\left(\boldsymbol{x}_{t}\right)\right)^{2} .
$$



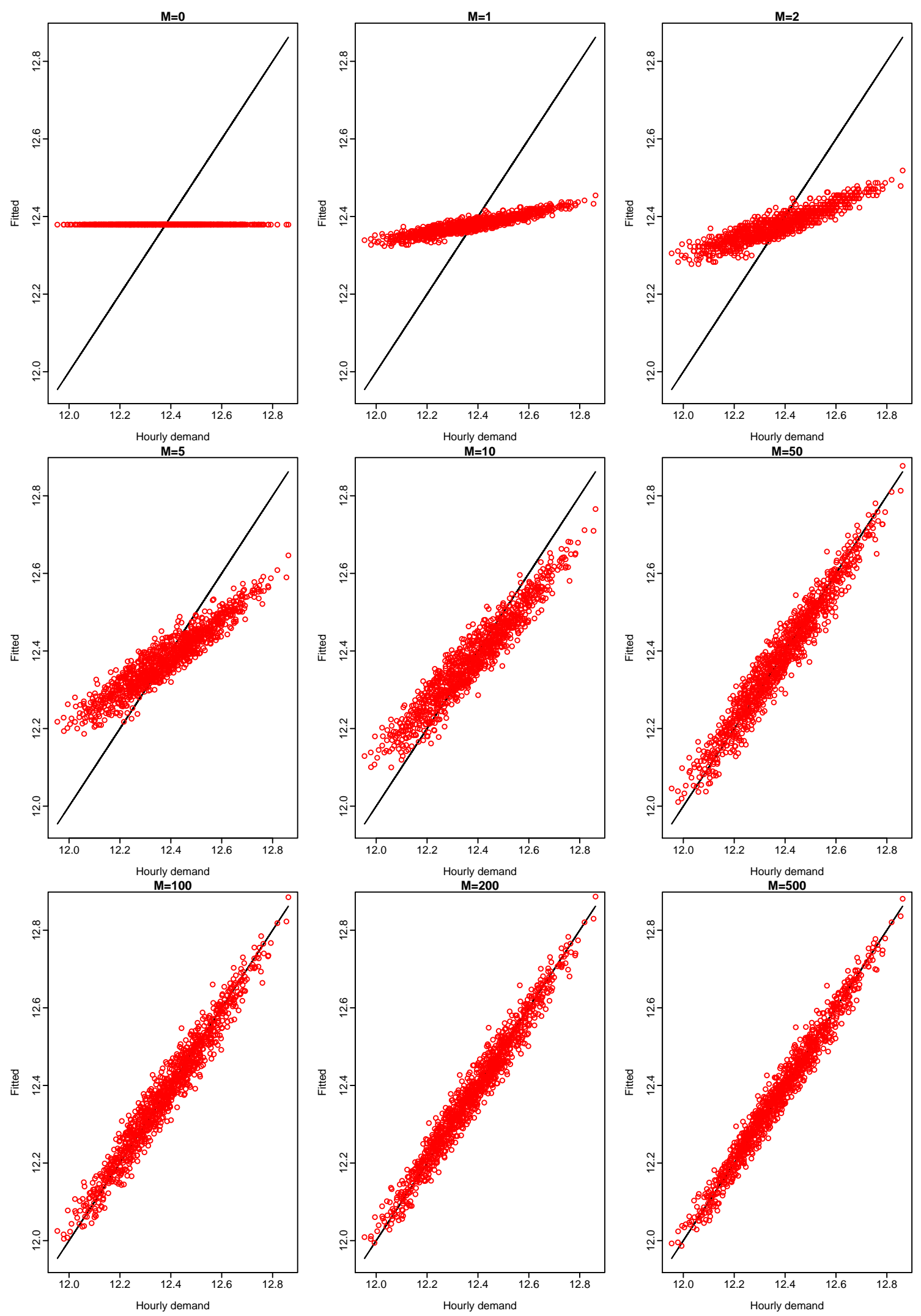

Figure 12: . $M=500$ is the best in terms of cross-validation. 
We then define the relative importance of a predictor $j$ as

$$
I_{j}=\frac{E^{(-j)}-E}{E}
$$

where $E$ is the squared error of the final model with all the selected variables. In other words, the predictors which most increase the error after removing their relative effect are the most influential variables given the other predictors.

Figure 13 shows the ten most influential variables (according to $I_{j}$ ) on the demand at different hours of the day. We assign the importance value $I_{j}=100$ to the most influential variable and the values of the others are scaled accordingly as in Friedman and Hastie (2000). To help visualisation, variables belonging to the same effect are plotted with the same color. That is, demand variables are colored in green, temperature variables in blue and calendar variables in red.

We can see that variables from the different effects are selected: calendar effect (in red), temperature effect (in blue) and lagged demand effect (in green). The importance of these different effects has been shown in Section 2. We have seen the different calendar effects in Figures 2-6. The clear dependence between demand and temperature was illustrated in Figure 7. Finally, Figure 8 has shown that there is a clear dependence between the actual demand and previous lagged demand variables.

For the first horizon $(h=1)$, we see that the most important variable is the current demand (variable 5). This is not surprising since the demand at hour $p+1$ is highly dependent on the demand at hour $p$. However, as one moves away from the starting point (i.e. $h=2-3$ ), other variables, such as the demand at the equivalent hour for the previous day (variable 17), become important while the current demand loses importance.

During working hours (10:00-19:00 or $h=4-13)$, temperature variables (in blue) dominate demand variables (in green). In fact, among the most important variables we find the current temperature with some of the corresponding lagged temperatures (variables 22--25). During that period of the day, we can also see that the variable 17 is gaining importance with the horizon, with the highest importance at 19:00.

For the last hours of the day (20:00-24:00 or $\mathrm{h}=14-18)$, temperature variables become the most influential variables. For 20:00 and 21:00, both variable 17 and 21 have a high importance while for the remaining hours of the day, only variable 17 remains as a most influential variable.

For the first hours of the next day (1:00-05:00), temperature variables are gaining more importance with a new variables appearing from 03:00 to 05:00, the average temperature of the previous day (variable 42).

Finally, at $06: 00$, we see that variable 17 is again the most influential variable together with the day of the week.

The analysis of Figure 13 has shown that the relative importance of the different variables and effects is changing with the time of the day. This shows that the dependence between the demand and the different considered variables is changing with the forecasting horizon, making electricity load forecasting a challenging statistical problem.

For illustration purposes, Figure 14 gives the forecasts of the aggregate series (i.e. the sum of the forecasts over all zones) for the eight in-sample weeks and the out-of-sample week.

\section{Conclusion}

Our entry ranked fifth out of 105 participating teams. This suggests that our modelling strategy is competitive with the other models used to forecast the electricity demand. We have identified several aspects that makes our modelling strategy successful.

First, as in any prediction task, data analysis allowed us to identify and clean the data from any corrupted information for better model performance. The data analysis step was also important for identifying useful variables to use in the model. 

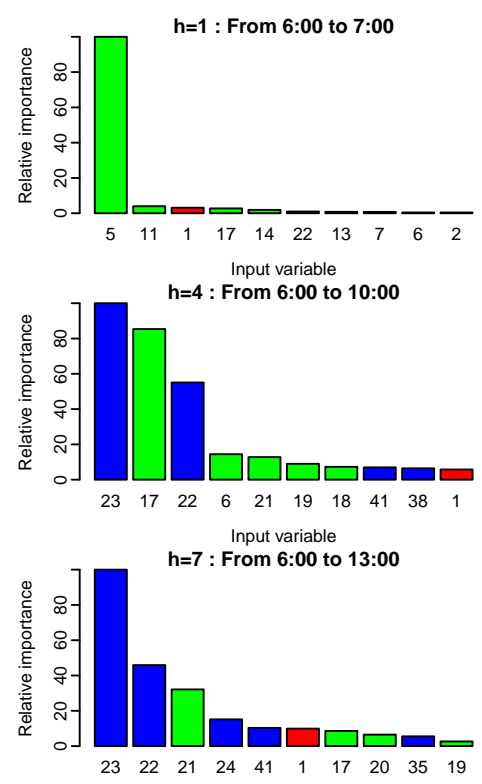

Input variable
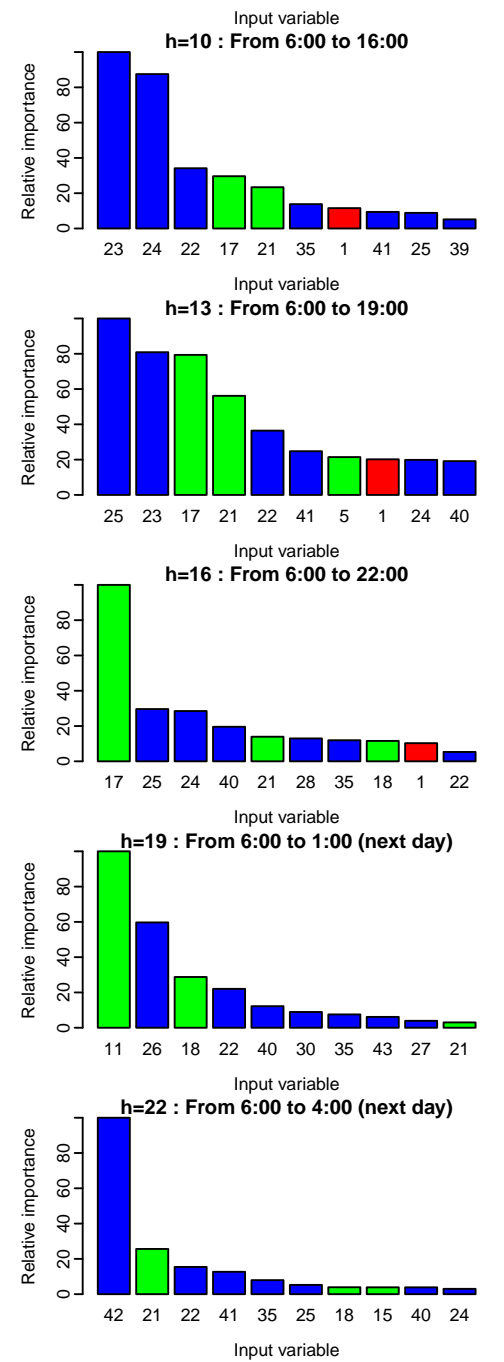
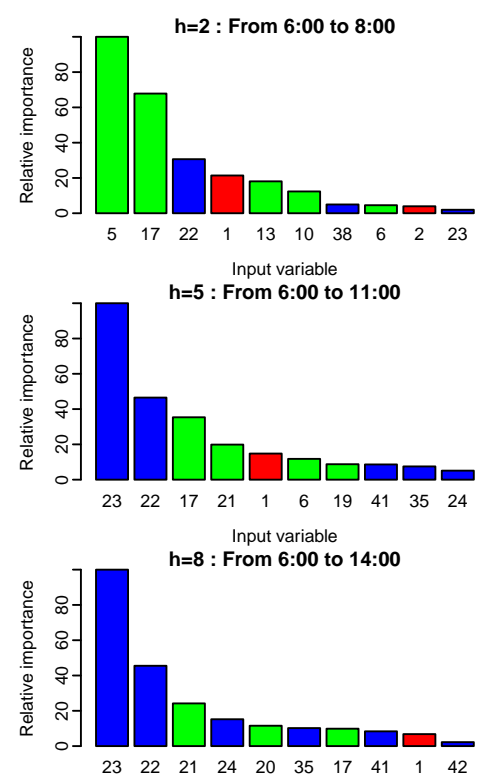

Input variable

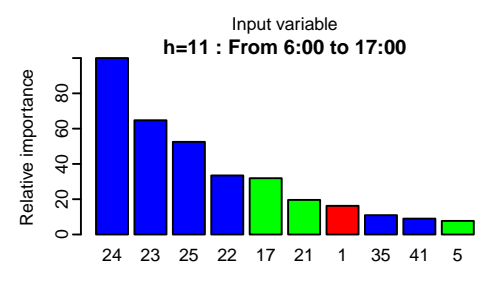

Input variable

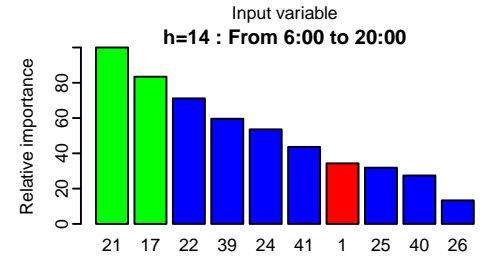

Input variable

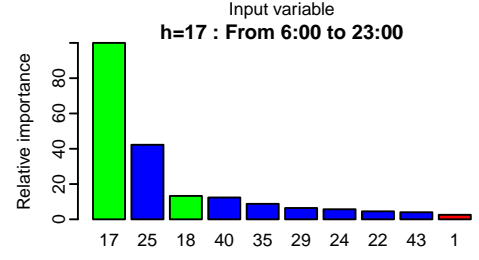

Input variable
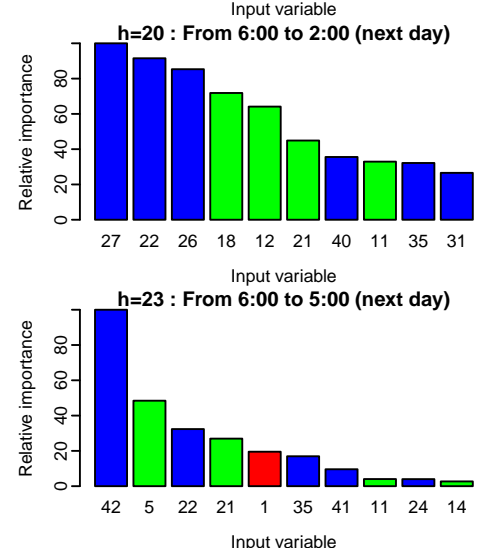
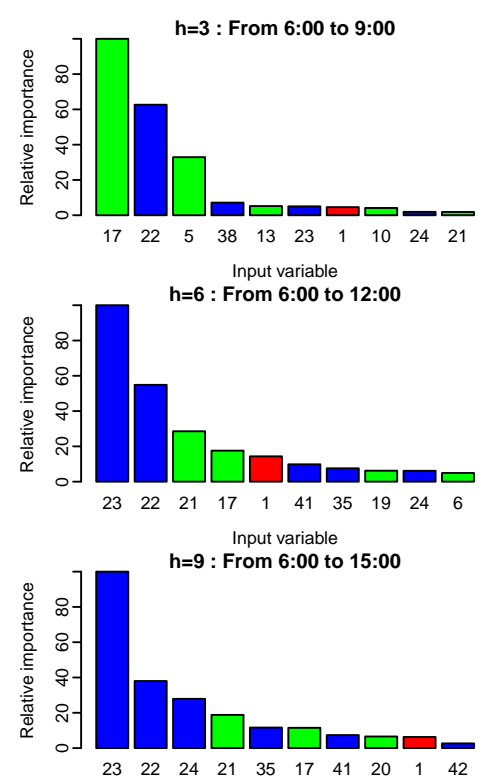

Input variable
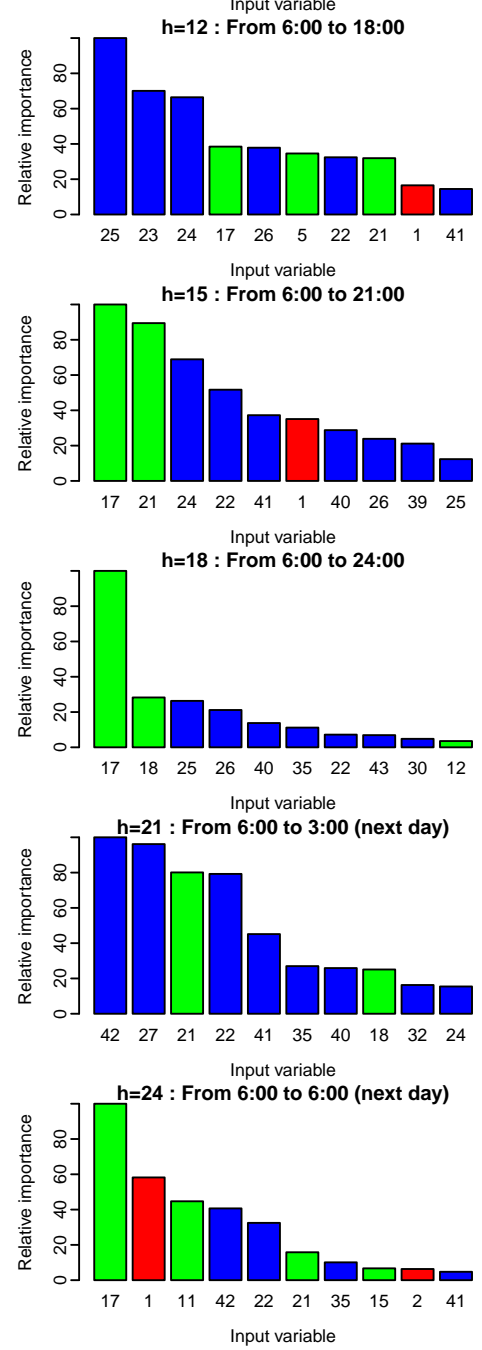

Figure 13: Relative importance of the five first variables on the demand for different times of the day. Demand variables are colored in green, temperature variables in blue and calendar variables in red. 

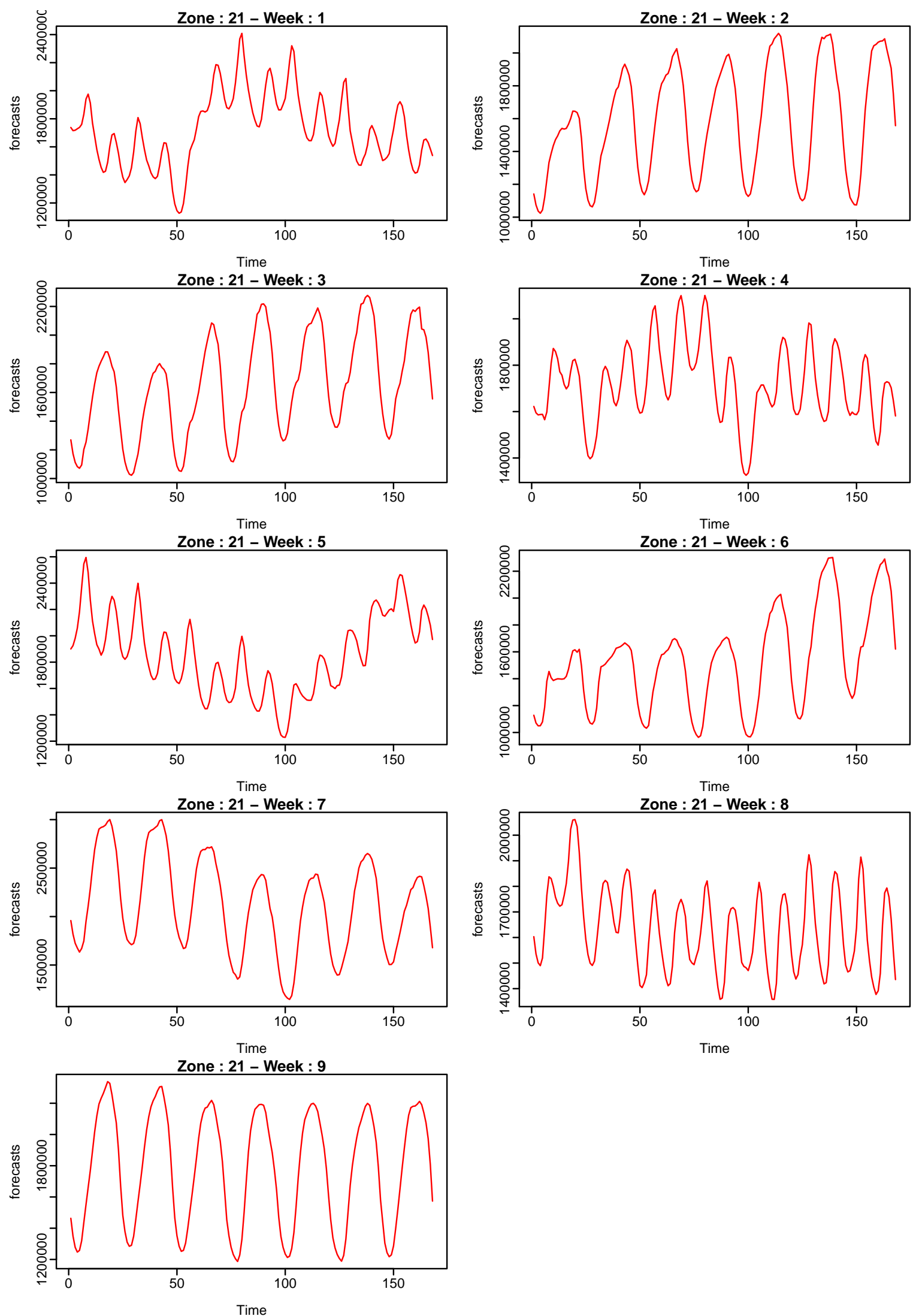

Figure 14: Forecasts for zone 21 for the eight in-sample weeks and the out-of-sample week. 
Second, we used different models including different effects for each hour of the day to model the demand patterns which are changing throughout the day.

Third, each hourly model allowed nonlinear and nonparametric terms to be included in the final model. This provided a great flexibility to the model and avoided making too many assumptions about the data generating process.

Finally, gradient boosting has proven many times to be an effective prediction algorithm for both classification and regression tasks. By selecting the number of components included in the model, we can easily control the so-called bias-variance trade-off in the estimation. In addition, componentwise gradient boosting increases the attractiveness of boosting by adding automatic variable selection during the fitting process.

The Kaggle load forecasting competition was a challenging prediction task which required solving several statistical problems such as data cleaning, variable selection, regression and multi-step time series forecasting.

\section{References}

Bühlmann, P. (2006), 'Boosting for high-dimensional linear models', Annals of Statistics 34(2), 559-583.

Bühlmann, P. and Hothorn, T. (2007), 'Boosting algorithms: Regularization, prediction and model fitting', Statistical Science 22(4), 477-505.

Bühlmann, P. and Yu, B. (2003), 'Boosting With the L2 Loss: Regression and Classification', Journal of the American Statistical Association 98, 324-339.

Eilers, P. H. C. and Marx, B. D. (1996), 'Flexible smoothing with B -splines and penalties', Statistical Science 11(2), 89-121.

Fan, S. and Chen, L. (2006), 'Short-Term Load Forecasting Based on an Adaptive Hybrid Method', IEEE Transactions on Power Systems 21(1), 392-401.

Fan, S. and Hyndman, R. J. (2012), 'Short-term load forecasting based on a semi-parametric additive model', IEEE Transactions on Power Systems 27(1), 134-141.

Fay, D., Ringwood, J. V., Condon, M. and Kelly, M. (2003), '24-h electrical load dataa sequential or partitioned time series?', Neurocomputing 55(3-4), 469-498.

Freund, Y. (1995), 'Boosting a weak learning algorithm by majority', Information and computation pp. 1-50.

Freund, Y. and Schapire, R. R. E. (1996), Experiments with a New Boosting Algorithm, in 'Proceedings of the Thirteenth International Conference on Machine Learning', pp. 148-156.

Friedman, J. H. (2001), 'Greedy function approximation: A gradient boosting machine.', Annals of Statistics 29(5), 11891232.

Friedman, J. and Hastie, T. (2000), 'Additive logistic regression: a statistical view of boosting (With discussion and a rejoinder by the authors)', Annals of Statistics 28(2), 337-407.

Hastie, T. J. and Tibshirani, R. (1995), Generalized additive models, Chapman \& Hall/CRC, London.

Hothorn, T., Bühlmann, P., Kneib, T., Schmid, M. and Hofner, B. (2012), mboost: Model-based boosting. R package version 2.1-3.

McSharry, P. E., Bouwman, S. and Bloemhof, G. (2005), 'Probabilistic forecasts of the magnitude and timing of peak electricity demand', IEEE Transactions on Power Systems 20, 1166-1172.

Mease, D. (2008), 'Evidence Contrary to the Statistical View of Boosting : A Rejoinder to Responses', Journal of Machine Learning Research 9, 195-201.

Mease, D. and Wyner, A. (2008), 'Evidence contrary to the statistical view of boosting', The Journal of Machine Learning Research 9, 131-156.

Ramanathan, R., Engle, R. F., Granger, C. W. J., Vahid, F. and Brace, C. (1997), 'Short-run forecasts of electricity loads and peaks', International Journal of Forecasting 13, 161-174.

Schapire, R. (1990), 'The strength of weak learnability', Machine learning 5(2), 197-227.

Schapire, R. E. and Freund, Y. (2012), Boosting: Foundations and Algorithms, The MIT Press.

Schapire, R. E., Freund, Y., Bartlett, P. and Lee, W. S. (1998), 'Boosting the margin: a new explanation for the effectiveness of voting methods', Annals of Statistics 26(5), 1651-1686.

Tao, H., Pierre, P. and Shu, F. (2013), 'Global Energy Forecasting Competition 2012', International Journal of Forecasting 29. this issue. 